\title{
Enhancing Surveillance on the BioSense Platform through Improved Onboarding Processes
}

\author{
Travis Mayo*1, Michael Coletta ${ }^{2}$, Sophia Crossen ${ }^{3}$ and Kirsten Oliver ${ }^{4}$ \\ IInductiveHealth Informatics, Atlanta, GA, USA; ${ }^{2}$ Centers for Disease Control and Prevention, Atlanta, GA, USA; ${ }^{3}$ Kansas Department \\ of Health and Environment, Topeka, KS, USA; ${ }^{4}$ West Virginia Department of Health and Human Resources, Charleston, WV, USA
}

\section{Objective}

This session will present the impacts of enhancements made to National Syndromic Surveillance Program (NSSP) BioSense Platform Onboarding in 2017 from the perspective of CDC and public health jurisdictions.

\section{Introduction}

In 2017, the National Syndromic Surveillance Program (NSSP) continued to expand as a national scope data source with over 6,500 facilities registered on the BioSense Platform, including 4,000 active, 1,800 onboarding, and 700 planned or inactive facilities. 2,086 of the active facilities are Emergency Departments across 49 sites in 41 states. The growth of data available in NSSP has been driven by continued enhancements to tools and processes used by the NSSP Onboarding Team. These enhancements help to rapidly integrate new healthcare facilities and onboard new public health sites in support of American Hospital Association (AHA) Emergency Department (ED) representativeness goals. Furthermore, with these improvements to the onboarding process, including the Master Facility Table update process and automated data validation reporting, NSSP has broadened stakeholder participation in the onboarding process.

\section{Keywords}

Onboarding; Quality; Validation; Automation; NSSP

\section{*Travis Mayo}

E-mail: tmg4@cdc.gov 